\title{
Prenatal diagnosis and pregnancy determination of sex chromosome aneuploidy screened by non- invasive prenatal testing from 52,453 unselected singleton pregnancies: a retrospective analysis of 4- year experience
}

\section{Xiaojin Luo}

The Genetics Laboratory, Longgang District Maternity \&Child Heathcare Hospital of Shenzhen City https://orcid.org/0000-0001-6882-4194

\section{Liang Hu}

The Genetics Laboratory, Longgang District Maternity \&Child Heathcare Hospital of Shenzhen City, Shenzhen, Guangdong Province,518172, China

\section{Yuanyuan Pei}

The Genetic Laboratory, Longgang District Maternity \&Child Heathcare Hospital of Shenzhen City

\section{Xiaoyi Cong}

The Genetics Laboratory, Longgang District Maternity \&Child Heathcare Hospital of Shenzhen City

Xiaoyi Liu

The Genetics Laboratory, Longgang District Maternity \&Child Heathcare Hospital of Shenzhen City Jing Chen

The Genetics Laboratory, Longgang District Maternity \&Child Heathcare Hospital of Shenzhen of City

Gaochi Li

The Genetics Laboratory, Longgang District Maternity \&Child Heathcare Hospital of Shenzhen City

\section{Lijuan Wen}

The Genetics Laboratory, Longgang District Maternity \&Child heathcare Hospital of Shenzhen City Jinxing Liu

The Genetics Laboratory, Longgang District Maternity \&Child Heathcare Hospital of Shenzhen City Jian Ran

The Genetics Laboratory, Longgang District Maternity \&Child Heathcare Hospital of Shenzhen City Fengxiang Wei ( $\square 33498842 @ q q . c o m)$

https://orcid.org/0000-0003-3802-5345 
Keywords: Non-invasive prenatal testing, Sex chromosomal aneuploidy, Prenatal diagnosis, pregnancy determination

Posted Date: January 20th, 2020

DOI: https://doi.org/10.21203/rs.2.21260/v1

License: (1) (i) This work is licensed under a Creative Commons Attribution 4.0 International License.

Read Full License 


\section{Abstract}

Objective

To estimate the positive predictive value (PPV) of fetal sex chromosome aneuploidy (SCA) screened in non-selective population and explore the rate of pregnancy termination and ultrasound findings for fetal SCA and the factors influencing parents' decisions in South China.

\section{Methods}

This is a large-scale retrospective cohort of positive SCA screened from unselected singleton pregnancies by non-invasive prenatal testing (NIPT) from a single prenatal center of a tertiary hospital, from January 2016 to November 2019. Clinical information, indications, diagnostic results, ultrasound findings, pregnancy determinations, and follow-up were reviewed and analyzed.

\section{Results}

248 cases of SCA positive were screened out of 52453 , giving a positive detection rate of $0.47 \%$. The majority of indications (42.7\%) were low-risk pregnancies. After genetic counseling, 43 pregnancies (17.3\%) declined to prenatal diagnosis, the rest of 205 cases (82.7\%) conducted with amniocentesis to detect fetal chromosome, of which 95 were confirmed as true positive of SCA with PPV of $46.3 \%$ $(95 / 205)$. The SCA consisted of 68 sex chromosomal trisomies (26 cases of $47, X X Y, 20$ cases of $47, X X X$ and 22 cases of $47, X Y Y), 17$ cases of monosomy $X(45, X)$, three cases of $48, X X Y Y$, three cases of mosaicisms $(45, X / 46, X X)$, four cases with sex chromosomal deletions, included two cases of $46, X$,del $(Y)$ (q11.21), one case of $46, X, \operatorname{del}(X)(p 11)$ and one case of $46, X, i(X)(q 10)$. Of the 95 cases confirmed as true positive SCA, 50 cases (52.6\%) chose to terminate the pregnancy $(82.6 \%, 64.3 \%, 17.6 \%$ and $33.3 \%$ for $45, X$, $47, X X Y, 47, X X X$ and $47, X Y Y$, respectively), 45 cases (47.4\%) elected to continue the pregnancy. Ninetythree pregnant were also continued pregnancy after the exclusion of SCA.

\section{Conclusions}

NIPT, as a first-tier routine method for screening autosomal aneuploidies, also could play an important role in screening SCA. Low-risk pregnant women are the main indication for the detection of SCA as NIPT test provides to non-selective population. The trend of overall termination rates of SCA-affected pregnancies is decreased. For 47,XXX and 47,XYY with mild phenotype, couples would like to continue the pregnancy. But for $45, X$ and $47, X X Y$, parents apt to terminate pregnancy no matter ultrasound abnormalities were found or not. pregnancy determinations are affected by types of SCA, sonographic findings, maternal age, and presence of infertility.

\section{Introduction}

Sex chromosomal aneuploidy (SCA), the incidence of newborn ranged from $1 / 1200$ to $1 / 400$. The incidence of fetal period is as high as $1 / 435[1,2]$. Common types are sex chromosome monosomy 
$(45, X)$, sex chromosome trisomy $(47, X X X, 47, X X Y$ and $47, X Y Y)$, other aneuploidies and Various forms of mosaic chromosomal abnormalities [3]. Unlike autosomal chromosome aneuploidies ( trisomy 21, trisomy 13 and trisomy 18), most of the SCA patients have very mild phenotype and relatively better clinical prognosis [4]. They can be manifested as abnormal height development, abnormal organ structure, abnormal behavior, dysplasia of secondary sexual characteristics and so on. The phenotype of SCA patients are diversities, and some patients have no abnormal phenotype throughout their lifetimes (47,XXX and 47,XYY) [5]. Therefore, These make prenatal counseling and parental decision-making following the prenatal diagnosis of SCA more complicated and challenging. Its pregnancy determination of SCA is often influenced by many factors, such as types of SCA, ultrasound findings, family's socioeconomic level, education level, religions, couple's understanding and acceptance of SCA. Therefore, Prenatal screening or diagnosis of SCA is not only challenged clinical genetic counseling but also puts pregnancy determination in a dilemma [6].

With the development of second-generation sequencing technology, non-invasive prenatal testing (NIPT) has been rapidly and widely used worldwide [7]. NIPT has higher sensitivity and specificity in screening trisomy 21, trisomy 18 and trisomy 13 syndromes [8]. It has been recommended by many professional associations of Obstetrics and gynecology as the most accurate prenatal screening method for aneuploidy $[9,10]$. However, the effectiveness of NIPT in detecting SCA and whether SCA should be included in the NIPT report as a routine report are still controversial. In addition to sensitivity and specificity, the positive predictive value (PPV) is also an important index in evaluating the detection ability of a screening method [11]. Previous reports suggested that the sensitivity of NIPT for SCA screening could reach $88.6 \% \sim 93.8 \%$, but PPV could only reach approximately $50 \%[4,6,12]$. The incidence of different types of SCA is very variable, and most of the previous studies have limited sample size, pregnancies with limited screening indications and the fluctuation range of PPV is various $[6,12]$. Hence, In the present retrospective study, we reviewed SCA cases that a large-scale and found in non-selective pregnancies who underwent NIPT. The results of prenatal diagnosis, ultrasound findings, distribution of indications, pregnancy determination and follow-up of SCA cases were analyzed, the PPV of positive SCA was calculated.

\section{Materials And Methods}

NIPT testing was provided for an almost free charge for all enrolled pregnant women with Public Healthcare Service and insurance coverage. All singleton pregnancies who underwent NIPT from January 2016 to November 2019 at the prenatal diagnosis center of Longgang district Maternity\&Child healthcare hospital of Shenzhen were selected, indications were included Low-risk population, advanced maternal age( $\geqq 35)$, high risk of serum screening, intermediate risk of serum screening, abnormal ultrasound findings and Increased NT (>2.5mm). Exclusion criteria include twin or more pregnancies, one of the couples has a clear chromosome number or structural abnormality. Pregnant women who have received an allogeneic blood transfusion, transplantation and stem cell therapy within one year are excluded. Clinical information on indications, results of invasive prenatal diagnosis, ultrasound findings, pregnancy 
determination, and follow-up were collected. This study was approved by the Hospital Ethics Committee and informed consent was obtained from each participant.

NIPT testing: Five $\mathrm{mL}$ of maternal peripheral blood was collected into an EDTA tube. Plasma was isolated from blood within 6 hours by two-step centrifugation, whole blood was centrifuged at $1600 \mathrm{~g}$ for 10 min at $4^{\circ} \mathrm{C}$, and supernatants were transferred to $2.0 \mathrm{~mL}$ tube continued to centrifuged at $16000 \mathrm{~g}$ for $10 \mathrm{~min}$ at $4^{\circ} \mathrm{C}$. The plasma was immediately stored at $-80^{\circ} \mathrm{C}$ until DNA extraction. Afterward, cffDNA extraction, library construction, quality control, and pooling were performed according to the manufacturer's protocol, Fetal Chromosome Aneuploidy Testing Kits (BGI-Wuhan, Hubei, China) was used to conduct large-scale parallel genome sequencing by BGIS EQ-500 sequencing platform ( BGI-Wuhan, Hubei, China).

Sequencing data were compared with the human genome reference sequence (HG19, NCBI build 36). The $z$-score for each chromosome was calculated to judge abnormality referencing to the normalized chromosome representation. Samples failing the quality criteria of cffDNA extraction, library construction, and sequencing as well as fetal DNA concentration (< $4 \%)$ were kicked out.

Invasive Prenatal diagnosis: Pregnancies associated with positive SCA were offered invasive prenatal diagnosis, fetal chromosomal karyotyping were analyzed. Amniotic Fluid samples were obtained according to the prenatal procedure protocol. Amniocytes were cultured with Gibco medium (Life Technologies Corporation, New York, United States). The cultured amniocytes were analyzed by routine cytogenetic analysis using G-banding techniques at a resolution of 400-500 bands. The number of metaphases examined varied between 20 and 30 . The results of cultured amniocytes are available within 14 to 20 days. Pregnancies who declined to have prenatal diagnosis were followed-up until delivered for at least 3 months.

Statistical methods: Descriptive statistics were applied, using the Chi-squared test when comparing discrete categorical variables. SPSS 20.0 software (SPSS Inc., Chicago, USA) was used to analyze all data for statistical significance. Data are presented as mean $\pm S D$ s. Analysis of variance was used to compare the differences between different groups. A $P$-value $<0.05$ was considered statistically significant.

\section{Results}

General information: From January 2016 to November 2019, 52,661 singleton pregnancies who underwent NIPT screening, 52,453 pregnancies were successfully got available results and 208 pregnancies were failed due to inadequate fetal fragments. Figure 1 shows the brief flow of this study. The median maternal age of the blood collection was $29.3 \pm 4.69$ years, ranging from 16 to 48 years. The median gestational weeks were $16.8 \pm 3.4$ weeks, varied from 12 to 34 weeks. There were $248(0.47 \%$, 248/52453) positive SCA screened, including 83 cases with 45,X, 63 cases of 47,XXY, 49 cases with 47,XXX and 53 cases with 47,XYY. Table 1 shows the clinical indications of pregnancies with positive SCA. Prenatal karyotyping was performed in $205(82.7 \%, 205 / 248)$ cases, 43 cases $(17.3 \%, 43 / 248)$ were declined to conduct prenatal diagnosis. No significant differences were found in the prenatal diagnostic rate of different kinds of positive SCA $\left(X^{2}=1.63, p>0.05\right)$. 95 cases were confirmed with true positive SCA, 
the overall PPV of positive SCA in this study was $46.3 \%(95 / 205)$, the PPV of $45, X, 47, X X Y, 47, X X X$, and $47, X Y Y$ were $32.9 \%, 51.8 \%, 58.3 \%$ and $51.2 \%$, respectively. The overall termination rate of true positive SCA was $52.6 \%$ (50/95), the termination rate of $45, X, 47, X X Y, 47, X X X$ and $47, X Y Y$ were $82.6 \%, 64.3 \%$, $17.6 \%$ and $33.3 \%$, respectively. Details are listed in Table 1 and Table 2.

Indications of positive SCA Cases: Among 248 cases, 106 pregnancies $(42.7 \%, 106 / 248)$ with low-risk before NIPT screening. 81 pregnancies $(32.7 \%, 81 / 248)$ with advanced maternal age( $\geqq 35) .19$ pregnancies $(7.7 \%, 19 / 248)$ with a high risk of serum screening (referring to Down's screening risk value $>1 / 270)$. 25 pregnancies $(10.1 \%, 25 / 248)$ with an intermediate risk of serum screening (referring to Down's screening risk value $1 / 1000-1 / 270) .12$ pregnancies $(4.8 \%, 12 / 248)$ with ultrasound abnormalities. 5 case $(2.0 \%, 5 / 248)$ with Increased NT(>2.5mm) .Table 1.

\begin{tabular}{|llllll|}
\hline \multicolumn{6}{|l|}{ Table 1. Indications of pregnancies with positive results of SCA screened by NIPT } \\
\hline Indications & $45, \mathrm{X}$ & $47, \mathrm{XXY}$ & $47, \mathrm{XXX}$ & $47, \mathrm{XYY}$ & Total \\
\hline Low risk prior to NIPT screening & 44 & 15 & 16 & 31 & $\begin{array}{l}106 \\
\text { Advanced maternal age( } \geqq 35)\end{array}$ \\
\hline High risk of serum screening & 12 & 32 & 26 & 11 & $81(32.7 \%)$ \\
\hline $\begin{array}{l}\text { Intermediate risk by serum } \\
\text { screening }\end{array}$ & 9 & 2 & 3 & 6 & $19(7.7 \%)$ \\
\hline Abnormal ultrasound findings & 6 & 11 & 3 & 2 & $25(10.1 \%)$ \\
\hline Increased NT (> 2.5 mm) & 4 & 2 & 1 & 3 & $12(4.8 \%)$ \\
\hline Total & 83 & 1 & 0 & 0 & $5(2.0 \%)$ \\
\hline
\end{tabular}


Table 2. Results of prenatal diagnosis and prenatal decisions of SCA in total 248 cases

\begin{tabular}{|c|c|c|c|c|c|c|c|}
\hline $\begin{array}{l}\text { Type } \\
\text { of SCA }\end{array}$ & $\mathrm{n}$ & $\begin{array}{l}\text { Cases of } \\
\text { prenatal } \\
\text { diagnosis. } \\
n\end{array}$ & $\begin{array}{l}\text { Rate of } \\
\text { prenatal } \\
\text { diagnosis. } \\
\%\end{array}$ & $\begin{array}{l}\text { Cases of } \\
\text { Ture } \\
\text { positive.n }\end{array}$ & $\begin{array}{l}\text { Positive } \\
\text { predictive } \\
\text { value \% }\end{array}$ & $\begin{array}{l}\text { Termination } \\
\text { of } \\
\text { pregnancy. } \\
\mathrm{n}(\%)\end{array}$ & $\begin{array}{l}\text { Continuation } \\
\text { of } \\
\text { pregnancy } \\
. n(\%)\end{array}$ \\
\hline $45, X$ & 83 & 70 & 84.3 & $23^{a b c}$ & 32.9 & 19 (82.6) & $4(17.4)$ \\
\hline $47, X X Y$ & 63 & 56 & 88.9 & $29^{d}$ & 51.8 & $22(64.3)$ & 7 (35.7) \\
\hline $47, X X X$ & 49 & 36 & 73.5 & $21^{e}$ & 58.3 & $3(17.6)$ & $18(82.4)$ \\
\hline $47, X Y Y$ & 53 & 43 & 81.1 & 22 & 51.2 & $6(33.3)$ & $16(66.7)$ \\
\hline Total & 248 & 205 & 82.7 & 95 & 46.3 & $50(52.6)$ & $45(47.4)$ \\
\hline
\end{tabular}

Prenatal diagnosis, Ultrasound findings and Pregnancy determinationロAfter genetic counseling, 43 pregnancies were refused confirmatory examination, 205 pregnancies conducted invasive prenatal diagnosis. Among 83 pregnancies received positive results for $45, X$, invasive prenatal diagnosis was conducted in 70 pregnancies. 17 cases were confirmed as true positive $45, X$; three cases were mosaic $45, X$; three cases were $X$ or $Y$ chromosomal deletions. The PPV of $45, X$ was $32.9 \%$. Among 17 cases were confirmed as $45, X, 11$ cases were showed abnormal ultrasound results (six cases of cystic hygromas, two cases of ambiguous genitalia, one case of pleural effusion, one case of heart defect and one case of hydrops in abdominal cavity), 5 cases with mosaic 45 , $X$ were showed no abnormal ultrasound results. The pregnancy determination and ultrasound findings are summarized in Table 2 and Table 3 . Three cases of mosaic $45, X$ were all chosen to continue due to low-level mosaicism. 16 out of 17 pregnancies with $45, X$ and three pregnancies with deletions of sex chromosomes were terminated.

Karyotyping analysis was available for 56 out of 63 NIPT results of 47,XXY (88.9\%). 26 cases were confirmed as $47, \mathrm{XXY}, 3$ cases were tetrasomy $(48, \mathrm{XXYY})$ and 27 cases were normal. The PPV of 47,XXY was $58.3 \%$. Of the 26 pregnancies with confirmed results, Two cases with abnormal ultrasonic results (1 case with NT $=4.3 \mathrm{~mm}, 1$ case with hyperechogenic bowel ), and 17 cases confirmed with 47,XXY and 3 cases with $48, X X Y Y$ were chosen to terminate pregnancy. For 36 out of 49 NIPT results of $47, X X X$ with prenatal diagnosis (73.5\%), 20 were confirmed as triple $X$ (one case ultrasound showed NT $=4.1 \mathrm{~mm}$ ), one case as deletion of $X$ chromosome and 15 cases with normal karyotyping. The PPV of $47, X X X$ was $58.3 \%$ and only two cases elected to terminate. Of pregnancies with positive screening for 47,XYY, karyotyping analysis was available in 43 cases (81.1\%). 22 cases were confirmed and ultrasound abnormalities were found in two cases (one case with ventricular septal defect and one case with aortic coarctation). the PPV of $47, \mathrm{XYY}$ was $51.2 \%$ and Six cases were chosen to terminate pregnancy. 


\begin{tabular}{|c|c|c|c|c|c|c|}
\hline $\begin{array}{l}\text { SCA } \\
\text { Positive } \\
\text { by NIPT }\end{array}$ & $\begin{array}{l}\text { Results of } \\
\text { prenatal } \\
\text { diagnosis }\end{array}$ & $\begin{array}{l}\text { Number } \\
\text { of } \\
\text { Cases. } \\
n\end{array}$ & $\begin{array}{l}\text { Absence of } \\
\text { Ultrasound } \\
\text { abnormalities } \\
. n\end{array}$ & $\begin{array}{l}\text { Presence of } \\
\text { Ultrasound } \\
\text { abnormalities.n }\end{array}$ & $\begin{array}{l}\text { Termination } \\
\text { of } \\
\text { pregnancy } \\
\text {.n }\end{array}$ & $\begin{array}{l}\text { Continuation } \\
\text { of } \\
\text { pregnancy .n }\end{array}$ \\
\hline \multirow[t]{5}{*}{$45, X$} & $46, \mathrm{XN}$ & 47 & 46 & 1 & 0 & 47 \\
\hline & $45, X$ & 17 & 5 & $12(70.6 \%)$ & 16 & 1 \\
\hline & $\begin{array}{l}\operatorname{mos} \\
45, X / 46, X N\end{array}$ & 3 & 3 & 0 & 0 & 3 \\
\hline & $\begin{array}{l}46, X, \operatorname{del}(X) \\
(\mathrm{p} 11)\end{array}$ & 1 & 0 & 1 & 1 & 0 \\
\hline & $\begin{array}{l}46, \mathrm{X}, \mathrm{del}(\mathrm{Y}) \\
(\mathrm{q} 11.21)\end{array}$ & 2 & 2 & 0 & 2 & 0 \\
\hline \multirow[t]{3}{*}{$47, X X Y$} & $46, X N$ & 27 & 27 & 0 & 0 & 27 \\
\hline & $47, X X Y$ & 26 & 24 & 2 & 17 & 9 \\
\hline & $48, X X Y Y$ & 3 & 3 & 0 & 2 & 1 \\
\hline \multirow[t]{3}{*}{$47, X X X$} & $46, \mathrm{XN}$ & 15 & 15 & 0 & 0 & 15 \\
\hline & $47, X X X$ & 20 & 19 & 1 & 2 & 18 \\
\hline & $\begin{array}{l}46, X, \mathrm{i}(\mathrm{X}) \\
(\mathrm{q} 10)\end{array}$ & 1 & 1 & 0 & 1 & 0 \\
\hline \multirow[t]{2}{*}{$47, X Y Y$} & $46, \mathrm{XN}$ & 21 & 21 & 0 & 0 & 21 \\
\hline & $47, X Y Y$ & 22 & 20 & 2 & 6 & 16 \\
\hline Total & & 205 & 187 & 19 & 50 & 45 \\
\hline
\end{tabular}

Pregnancies decline to conduct prenatal diagnosis: Among 248 cases with positive SCA by NIPT, 43 pregnancies declined amniocentesis to confirm fetal chromosome. 31 cases had a live birth and no visible abnormal phenotype was found in clinical follow-up, 3 cases were still continued pregnancy with normal ultrasound findings. Two cases with positive results for $45, \mathrm{X}$ were terminated the pregnancy due to ultrasound abnormalities (one for Cystic hygroma and one for pleural effusion). Three cases with positive results (two cases for $45, \mathrm{X}$ and one case for $47, \mathrm{XXX}$ ) had spontaneous abortions in the second trimester. Besides, four cases lost to follow up after NIPT screening. The follow-up information was described in Table 4. 
Table 4. The follow-up information of $\mathbf{4 3}$ cases positive pregnancies without prenatal diagnosis

\begin{tabular}{|c|c|c|}
\hline Type of SCA & Prenatal decisions and follow-up information & Cases. $\mathrm{n}$ \\
\hline \multirow[t]{5}{*}{$45, x$} & Terminate based on ultrasound abnormalities & 2 \\
\hline & Spontaneous abortion in gestation & 2 \\
\hline & No visible abnormality in follow up & 5 \\
\hline & Postnatal testing as normal karyotype $46, \mathrm{XX}$ & 2 \\
\hline & Lost to follow up & 2 \\
\hline \multirow[t]{2}{*}{$47, X X Y$} & No visible abnormality in follow up & 5 \\
\hline & Lost to follow up & 2 \\
\hline \multirow[t]{2}{*}{$47, X X X$} & Spontaneous abortion in gestation & 1 \\
\hline & No visible abnormality in follow up & 12 \\
\hline $47, X Y Y$ & No visible abnormality in follow up & 10 \\
\hline Total & & 43 \\
\hline
\end{tabular}

\section{Discussion}

NIPT as a prenatal screening method for autosomal aneuploidy has been widely applied clinical practice with superior detection and lower false-positive rates than conventional screening tests $[13,14]$. But the NIPT used to screening for SCA has been controversial because of less accurate compare to trisomies of 21,13 and 18 [15]. Few studies reported that some of SCA may with normal ultrasound findings and fertility but lead to probably abusive termination of pregnancy, believed that NIPT should be limited to phenotypically severe diseases $[16,17]$. In this study, base on a large-scale databases from unselected pregnancies we found the positive rate of SCA screening was $0.47 \%(248 / 52453)$, the overall PPV of SCA was $46.3 \%$ (32.9\%, 51.8\%, 58.3\% and $51.2 \%$ for $45, X, 47, X X Y, 47, X X X$ and $47, X Y Y$, respectively). Similar to previous studies that showed the positive rate of SCA screening was varied from $0.21-0.63 \%$, the overall PPV of SCA ranged from 32.2-54.5\% from selective pregnancies with relevant indications $[3,6,12,18]$. The reasons why the PPV for $45, \mathrm{X}$ was lower compared to other types of SCA, firstly, it could partially be caused by a highly variable amplification of chromosome $\mathrm{X}$ because of its lower Guanosine-Cytosine content [19]. Secondly, study showed that there is an age-related loss of chromosome $X$ in white cells in normal pregnancies, which influenced the effectiveness in predicting fetal 45,X.[20]. In our cohort, pregnancies with positive screening for a SCA who conducted prenatal diagnosis were higher $(82.7 \%)$ than other reports $[3,21]$, This difference may be caused by parental concerns about having a child with infertility and high risks of lack secondary sexual characteristics and gynecomastia in mainland China [12]. In contrary to Aarti er al reported that pregnancies with positive NIPT for a SCA elect prenatal diagnosis was only $34.3 \%(46 / 134)$, because of the relatively mild or moderate prognoses associated with 
liveborn infants with SCA compared to the invasive procedural-related risks and anxiety, the parental attitude is more likely to accept an SCA fetus [3].

Before NIPT applied to screen fetal SCA, that the detection of SCA is usually incidental during screening for Down syndrome by conventional screening methods [22, 23]. Previous studies had shown the distribution of indications for prenatal diagnosis for each SCA-affected pregnancy included ultrasonography findings( such as cystic hygroma and hydrops), advanced maternal age( $\geq 35$ years), positive of serum screening test, increased nuchal translucency and so on [6, 24]. For prenatal diagnosis of $45, X$ (Turner Syndrome), the majority of indications were fetal ultrasound abnormalities [6, 12]. For the other types of SCA, the main indications for prenatal testing were advanced maternal age and high-risk serum screening tests $[6,12]$. In the present study, our databases indicated that after NIPT was applied to screening for all enrolled pregnancies as a first-tier routine method with cost-effective by public healthcare service and insurance coverage, the charge for NIPT screening was almost free for every pregnant woman. Therefore, The distribution of indications for screening a positive SCA were sharply changed, the majority of indications (42.7\%) were low-risk population who before NIPT screening, no matter for screening $45, \mathrm{X}$ or other types of SCA. Furthermore, the prenatal diagnosis of SCA had become more common and the demand for adequate pre-test and post-test genetic counseling was increased considerably.

In this study, The majority of the abnormal ultrasound findings were found in cases of $45, \mathrm{X}(70.6 \%$, $12 / 17)$, included cystic hygromas, ascites, pleural effusion, heart defects, and ambiguous genitalia. For the other types of SCA $(47, X X Y, 47, X X X$ and $47, X Y Y)$ which ultrasound findings were not typically expected $(7.0 \%, 5 / 71)$ and relatively manifested mild phenotypes, such as NT thickened and heart defects. Demonstrated for $45, \mathrm{X}$ were more likely to be found ultrasound abnormalities than sex chromosomal trisomies. The previous study showed that obvious fetal ultrasound abnormalities had a highly significant impact on the parental decision, pregnancies prefer to terminate than those with normal ultrasound findings $[3,6,24,25]$. This was also shown in our study, that especially for $45, X$ with abnormal ultrasound and poor prognosis associated who were all elected to terminated pregnancy. However, based on our data, ultrasound findings were not the only reason for couples to decide whether to continue or terminate pregnancy, younger maternal age, absence of history infertility also significantly associated with termination decisions. For cases with $45, \mathrm{X}$ and $47, \mathrm{XXY}$ would like to terminate pregnancy even those with normal ultrasound findings. For 47,XXX and 47,XYY would like to continue pregnancy even those with ultrasound abnormalities, such as ventricular septal defect and aortic coarctation [12].

The termination rates of SCA were varied across different countries and the possible reasons are different types of SCA, absence or presence of ultrasound abnormality, cultural background, education level, religious and counseling practices $[6,26]$. According to our data, the overall termination rate was $52.6 \%$ (50/95) $(82.6 \%, 64.3 \%, 17.6 \%$ and $33.3 \%$ for $45, X, 47, X X Y, 47, X X X$ and $47, X Y Y$, respectively), the termination rates of this study were much lower than previous studies. Liao et al reported that the overall termination rates of SCA were $96 \%$ from 1990 to 2006 and $84 \%$ from 2010 to 2012 [27]. This may be during that period, the one-child policy to control the population growth and termination of pregnancy is 
legal to perform even up to the third trimester, suggested that may have encouraged couples to terminate pregnancy if exist a potential sexual development and physical problems in mainland China. Similar to Po et al reported that termination of abnormal pregnancies is illegal after 24 weeks in Hong Kong, that the overall termination rate of pregnant women with SCA was 55.6\% [6]. Studies also reported declining trends of termination decision of SCA-affected pregnancies that all authors believed, this trend was the consequence of more publications from unselected newborn and incidental prenatal diagnosed SCA, which results have shown more balanced information about the prognosis of SCA compared to biased information from skewed studies in the past $[3,6,12,28]$. Our study is consistent with those previously reports that the termination rate of SCA has declined and parents have a relatively optimistic attitude to accept a child with SCA. Past studies showed that pregnant women with a history of infertility were more likely to continue their affected pregnancies $[29,30]$. This may influenced by the difficulty of getting pregnant and desire for parenthood, which increases parents' tolerance for a child with SCA. Younger maternal age was associated with the decision to terminate SCA-affected pregnancies. According to our data, pregnancies with diagnoses of $45, X$ and $47, X X Y$ were terminated more often than those with diagnoses of $47, X X X, 47, X Y Y$ and mosaicism. This finding was also reported in other studies $[6,12]$, compared with 47,XXX, 47,XYY and mosaicism, 45,X and 47,XXY have more severe phenotypes, including abnormal sexual development, infertility, and behavioral problems. Mosaicism generally has variable phenotypes and mild prognosis according to the different levels of mosaic [31].

This study we collected the NIPT results, distribution of indications, results of prenatal diagnosis, ultrasound findings and determination of pregnancies based on a large size data from non-selective pregnancy women. We found out the higher rate of prenatal diagnosis, the change of indications prior to NIPT, the declining trend of termination rate as well as PPV for each SCA by NIPT, However, the limitation of this study is that can not follow-up all false-positive cases, the existence of CPM can not be ruled out. Most of SCA cases can not be diagnosed until childbearing age, and some of them can not be diagnosed throughout lifetimes [32]. Therefore, the sensitivity, false-positive rate and false-negative rate of NIPT screening for SCA can not be accurately calculated. Furthermore, There was a insufficient of postnatal data for those who elected to continue pregnancies but delivered at normal phenotype without karyotyping results. Due to the majority of pregnancies may feel that confirmatory testing is unnecessary if no abnormalities are detected at birth. The limitations of NIPT should be carefully explained before and after testing.

\section{Conclusion}

This study based on a large-scale sample screened for SCA from non-selective singleton pregnancies, that NIPT provided as a first-tier routine screening method for all enrolled pregnancies, the demand for prenatal counseling and diagnosis of SCA were increased sharply. Our data indicated that SCA can effectively screened by NIPT, especially in predicting sex chromosome trisomies. ultrasound abnormalities were closely associated with 45,X. Pregnancies with 45,X and 47,XXY were more likely elected to terminated than those with $47, X X X$ and $47, X Y Y$. Our data also highlighted the necessity for pre-and posttest counseling and prenatal diagnosis before chose to terminate pregnancy. Early screening and 
diagnosis of SCA may provide an opportunity for early intervention and comprehensive postnatal management, improving the life quality of the affected child. Our findings may provide more references for clinical genetic counseling and pregnancy management.

\section{Declarations}

\section{Funding}

The study was supported by funding for the training of high-level health professionals in the Project supported by Shenzhen Committee of Science and Technology. (Grant No. JCYJ20180305125647151). Longgang Science and Technology Development Foundation (Grant No.LGKCYLWS2019000791) and Longgang Key Laboratory of Birth Defects Prevention (Grant No.LGKCZSYS2018000010).

\section{Authors' contributions}

All authors have materially participated in this study and manuscript preparation. Xiaojin Luo, Liang Hu, Yuan-yuan Pei, Xiao-yi Cong, Xiao-yi Liu, and Jing Chen collected all clinical data and carried out all the molecular and cytogenetic analyses. Gao-chi Li and Li-juan Wen participated in the data analysis and drafted the manuscript. Jin-xing Liu and Jian Ran participated in the molecular genetic analyses. Fengxiang Wei designed the work and drafted and revised the manuscript. All authors have approved the final article.

\section{Availability of data and materials}

Please contact author for data requests.

\section{Ethics approval and consent to participate}

This study was approved by the Ethics Committee of Longgang District Maternity \&Child Heathcare Hospital of Shenzhen City, Shenzhen, Guangdong Province 518172, China. All participants gave written informed consent to take part in the present study.

\section{Consent for publication}

Consent for publication is not applicable.

\section{Competing interests}

The authors declare that they have no competing interests.

\section{References}

[1]Christian SM, Koehn D, Pillay R, et al. Parental decisions following prenatal diagnosis of sex chromosome aneuploidy: a trend over time. Prenat Diagn. 2000;20(1):37-40. 
[2]Nielsen J, Wohlert M. Sex chromosome abnormalities found among 34,910 newborn children: Results from a 13-year incidence study in Arhus, Denmark. Birth Defects Orig Artic Ser.1990; 26: 209-223.

[3]Ramdaney A, Hoskovec J, Harkenrider J, et al. Clinical experience with sex chromosome aneuploidies detected by noninvasive prenatal testing (NIPT): Accuracy and patient decision-making. Prenat Diagn. 2018;38(11):841-848.

[4]Linden MG, Bender BG, Robinson A, et al. Genetic counseling for sex chromosome abnormalities[J]. Am J Med Genet. 2002; 110(1):3-10.

[5]Ottesen AM, Aksglaede L, Garn I, et al. Increased number of sex chromosomes affects height in a nonlinear fashion: a study of 305 patients with sex chromosome aneuploidy. Am J Med Genet A. 2010;152A(5):1206-1212.

[6]So PL, Cheng KYY, Cheuk KY, et al. Parental decisions following prenatal diagnosis of sex chromosome aneuploidy in Hong Kong. J Obstet Gynaecol Res. 2017;43(12):1821-1829.

[7] Hu H, Wang L, Wu J, et al. Noninvasive prenatal testing for chromosome aneuploidies and subchromosomal microdeletions/microduplications in a cohort of 8141 single pregnancies. Hum Genomics. 2019;13(1):14.

[8]Chitty LS, Lo YM. Noninvasive Prenatal Screening for Genetic Diseases Using Massively Parallel Sequencing of Maternal Plasma DNA. Cold Spring Harb Perspect Med. 2015;5(9):a023085.

[9]Ronzoni L, Silibello G, Quagliarini D, et al. A comment on "clinical experience with sex chromosome aneuploidies detected by noninvasive prenatal testing (NIPT): Accuracy and patient decision-making". Prenat Diagn. 2018;38(13):1129-1130.

[10]Gregg AR, Skotko BG, Benkendorf JL et al. Noninvasive prenatal screening for fetal aneuploidy, 2016 update: A position statement of the American College of Medical Genetics and Genomics. Genet Med. 2016;18:1056-1065.

[11]Kalafat E, Seval MM, Turgay B, et al. Non-invasive prenatal testing for sex chromosome abnormalities: a source of confusion. BMJ Case Rep. 2015;18(3): 62.

[12]Xu Y, Chen L, Liu Y, et al. Screening, prenatal diagnosis, and prenatal decision for sex chromosome aneuploidy. Expert Rev Mol Diagn. 2019;19(6):537-542.

[13]Chen Y, Yu Q, Mao X, et al. Noninvasive prenatal testing for chromosome aneuploidies and subchromosomal microdeletions/microduplications in a cohort of 42,910 single pregnancies with different clinical features. Hum Genomics. 2019;13(1):60.

[14]Kim SY, Lee SM, Jun JK, et al. Prospective observations study protocol to investigate costeffectiveness of various prenatal test strategies after the introduction of noninvasive prenatal testing. 
BMC Pregnancy Childbirth. 2018;18(1):307.

[15]Jones KJ, Wang E, Bogard P, et al. Targeted cell-free DNA analysis with microarray quantitation for assessment of fetal sex and sex chromosome aneuploidy risk. Ultrasound Obstet Gynecol. 2018;51(2):275-276.

[16]Taneja PA, Snyder HL, De Feo E, et al. Noninvasive prenatal testing in the general obstetric population: clinical performance and counseling considerations in over 85000 cases. Prenat Diagn. 2016;36(3):237243.

[17]Gekas J, Langlois S, Ravitsky V, et al. Non-invasive prenatal testing for fetal chromosome abnormalities: review of clinical and ethical issues. Appl Clin Genet. 2016;9:15-26.

[18]Zhang B, Lu BY, Yu B, et al. Noninvasive prenatal screening for fetal common sex chromosome aneuploidies from maternal blood. J Int Med Res. 2017;45(2):621-630.

[19]Nicolaides KH, Musci TJ, Struble CA, et al. Assessment of fetal sex chromosome aneuploidy using directed cell-free DNA analysis. Fetal Diagn Ther. 2014;35(1):1-6.

[20]Russell LM, Strike P, Browne CE, et al. X chromosome loss and aging. Cytogenet Genome Res. 2007;116(3):181-185.

[21]García-Acero M, Moreno-Niño O, Suárez-Obando F, et al. Disorders of sex development: Genetic characterization of a patient cohort published online ahead of print. Mol Med Rep. 2019;21(1):97-106.

[22]Berglund A, Viuff MH, Skakkebæk A, et al. Changes in the cohort composition of turner syndrome and severe non-diagnosis of Klinefelter, 47,XXX and 47,XYY syndrome: a nationwide cohort study. Orphanet J Rare Dis. 2019;14(1):16.

[23]Pieters JJ, Kooper AJ, van Kessel AG, et al. Incidental prenatal diagnosis of sex chromosome aneuploidies:Health, behavior, and fertility. ISRN Obstet Gynecol. 2011; 20:106.

[24]Gil MM, Quezada MS, Revello R, et al. Analysis of cell-free DNA in maternal blood in screening for fetal aneuploidies: Updated meta-analysis. Ultrasound Obstet Gynecol.2015; 45: 249-266.

[25]Van Rijn S. A review of neurocognitive functioning and risk for psychopathology in sex chromosome trisomy (47,XXY, 47,XXX, 47, XYY). Curr Opin Psychiatry. 2019;32(2):79-84.

[26]Nishiyama M, Sekizawa A, Ogawa K,et al. Factors affecting parental decisions to terminate pregnancy in the presence of chromosome abnormalities: a Japanese multicenter study. Prenat Diagn. 2016;36(12):1121-1126.

[27]Liao C, Li DZ. Pregnancy outcome following prenatal diagnosis of sex chromosome abnormalities in Mainland China. Prenat Diagn 2008; 28:443-444. 
[28]Suzumori N, Kumagai K, Goto S, et al. Parental decisions following prenatal diagnosis of chromosomal abnormalities: implications for genetic counseling practice in Japan. J Genet Couns. 2015;24(1):117-121.

[29]Kim YJ, Park SY, Han JH, et al. Parental decisions of prenatally detected sex chromosome abnormality. J Korean Med Sci. 2002;17(1):53-57.

[30]Snyder HL, Curnow KJ, Bhatt S, et al. Follow-up of multiple aneuploidies and single monosomies detected by noninvasive prenatal testing: implications for management and counseling. Prenat Diagn. 2016;36(3):203-209.

[31]Wilson AC, King J, Bishop DVM, et al. Autism and social anxiety in children with sex chromosome trisomies: an observational study. Wellcome Open Res. 2019;4(6):32.

[32]Chai H, DiAdamo A, Grommisch B, et al. A Retrospective Analysis of 10-Year Data Assessed the Diagnostic Accuracy and Efficacy of Cytogenomic Abnormalities in Current Prenatal and Pediatric Settings. Front Genet. 2019;10(3):1162.

\section{Figures}




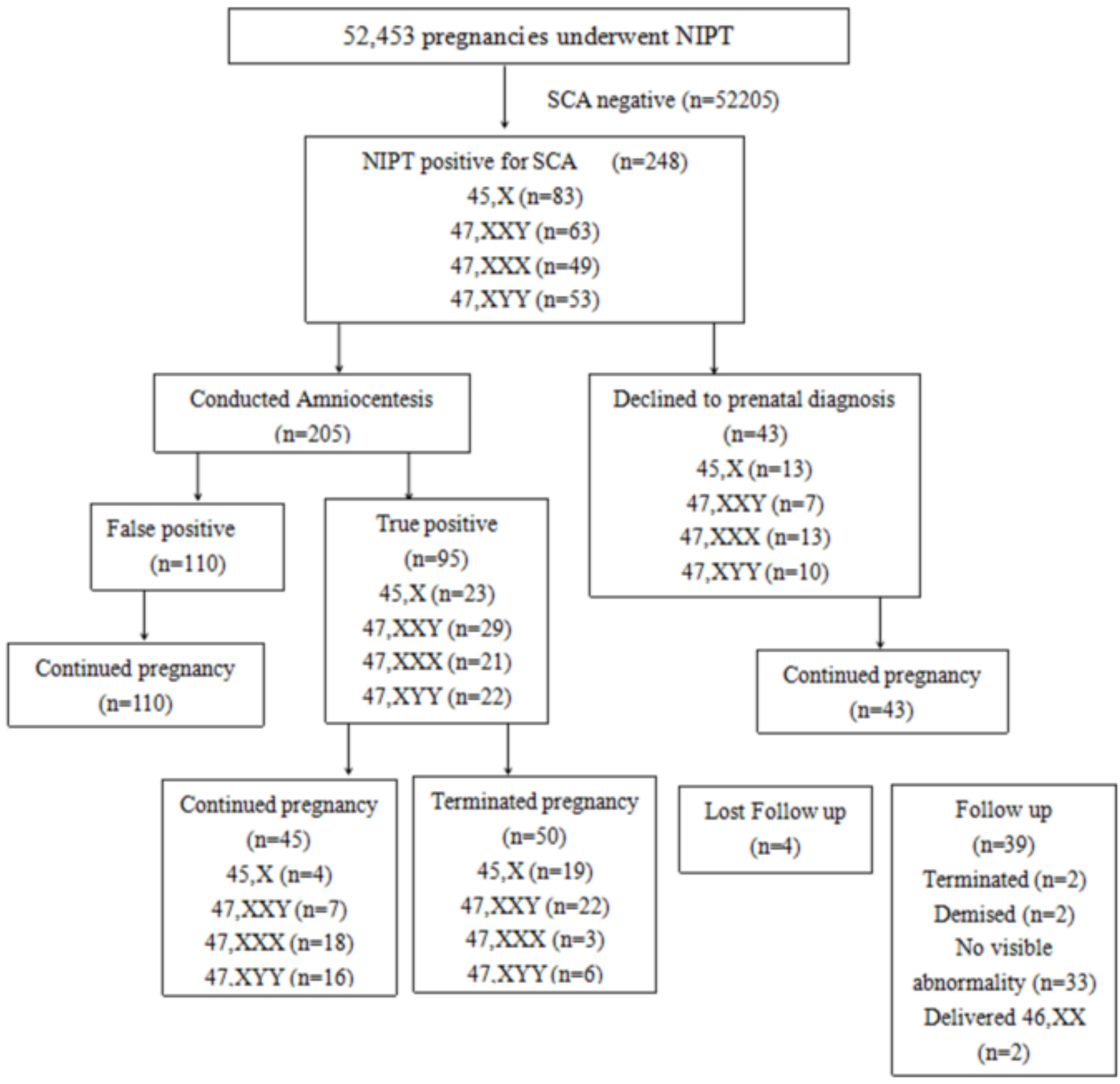

\section{Figure 1}

Study flow chart showing prenatal diagnosis and pregnancy determination of SCA positive screened by NIPT 